\title{
A common and two novel GBA mutations in Thai patients with Gaucher disease
}

\author{
Rachaneekorn Tammachote ${ }^{1,2}$, Siraprapa Tongkobpetch ${ }^{2}$, Chalurmpon Srichomthong ${ }^{3}$, Kampon \\ Phipatthanananti $^{2}$, Suthipong Pungkanon ${ }^{4}$, Duangrurdee Wattanasirichaigoon ${ }^{5}$, Kanya Suphapeetiporn ${ }^{2,3}$ \\ and Vorasuk Shotelersuk ${ }^{2,3}$
}

Gaucher disease (GD) is an autosomal recessive disorder caused by mutations in the glucocerebrosidase (GBA) gene, leading to a deficiency of lysosomal $\beta$-glucosidase and accumulation of glycosphingolipids in macrophages. We studied five Thai families with GD (four with GD type 1 and one with GD type 2). Using long-template PCR, PCR using specific primers for the functional gene, direct sequencing of all coding regions of GBA and restriction enzyme digestions, all 10 mutant alleles were successfully identified. The common c.1448T > C (p.L483P or L444P) mutation was identified in $60 \%$ of mutant alleles. Of the two patients homozygous for the p.L483P (L444P) mutation, one died from hepatic failure at age 16 years and the other died from sepsis at age 12 years. This p.L483P (L444P) mutation was found in four different haplotypes, suggesting that it was a recurrent mutation, not caused by a founder effect. Two novel mutations, a missense (c.1204T >C, p.Y402H), and a termination codon mutation (c.1609T>C, p.X537A) were found. Studies to determine the molecular pathomechanism of the p.X537A mutation, the first of its kind in this gene, showed that it decreased the amount of protein being expressed and the enzymatic activity, while it was still correctly localized.

Journal of Human Genetics (2013) 58, 594-599; doi:10.1038/jhg.2013.60; published online 30 May 2013

Keywords: Gaucher disease; GBA; mutation spectrum; termination codon mutation

\section{INTRODUCTION}

Gaucher disease (GD; MIM 230800), the most prevalent lysosomal storage disorder, is caused by a deficiency of lysosomal enzyme glucocerebrosidase, or acid $\beta$-glucosidase, which normally hydrolyzes glucocerebroside to glucose and ceramide, leading to accumulation of glucocerebroside in macrophages. There are three clinical subtypes of $\mathrm{GD}$, distinguished by the absence or presence and severity of neurologic complications. Type 1 (MIM 230800), or non-neuronopathic disease, does not involve the nervous system. Type 2 (MIM 230900), or acute-neuronopathic disease, is a fatal neurodegenerative disorder of infancy. Type 3 (MIM 231000), or chronic-neuronopathic disease, is a slowly progressive neurologic disease with survival into adulthood. Hepatosplenomegaly and bone lesions occur in all forms. ${ }^{1}$

The disease is an autosomal recessive disorder resulting from mutations in the glucocerebrosidase gene (GBA, MIM 606463) of $\sim 7.5 \mathrm{~kb}$, located on chromosome 1q21 and composed of 11 exons. A highly homologous $\sim 5.5 \mathrm{~kb}$ pseudogene (GBAP, MIM 606463) that shares $96 \%$ exonic sequence homology is located $16 \mathrm{~kb}$ downstream from the functional gene. ${ }^{2}$
At least 291 different mutations have been identified in the GBA gene (the Human Genome Mutation Database, http:// www.hgmd.cf.ac.uk/ac/index.php, February, 2013). The frequencies of specific mutated alleles vary in different populations. The most common mutation in the Ashkenazi Jewish, accounting for $\sim 70 \%$ of mutations is p.N409S (N370S). ${ }^{3}$ In this report, we studied the clinical, biochemical and molecular characteristics of five Thai families with GD.

\section{MATERIALS AND METHODS}

Subjects

Five unrelated Thai patients were diagnosed as having GD through clinical findings including hepatosplenomegaly and the presence of Gaucher cells in bone marrow aspiration. Based on the clinical findings, four unrelated patients were classified as type 1 and one as type 2. Consanguinity was denied in all families. With the exception of patient 1 , who had an elder brother with similar clinical features and died at the age 18 months, others were sporadic cases. Glucocerebrosidase activity in leukocytes was determined by using a mixture of 4-methyl-umbelliferone- $\beta$-D-glucopyranoside as a substrate and

${ }^{1}$ Department of Botany, Faculty of Science, Chulalongkorn University, Bangkok, Thailand; ${ }^{2}$ Department of Pediatrics, Center of Excellence for Medical Genetics, Faculty of Medicine, Chulalongkorn University, Bangkok, Thailand; ${ }^{3}$ Excellence Center for Medical Genetics, King Chulalongkorn Memorial Hospital, The Thai Red Cross Society, Bangkok, Thailand; ${ }^{4}$ Queen Sirikit National Institute of Child Health, Bangkok, Thailand and ${ }^{5}$ Department of Pediatrics, Faculty of Medicine, Ramathibodi Hospital, Mahidol University, Bangkok, Thailand

Correspondence: Professor Kanya Suphapeetiporn, Division of Medical Genetics and Metabolism, Department of Pediatrics, King Chulalongkorn Memorial Hospital, Sor Kor Building 11th floor, Bangkok 10330, Thailand.

E-mail: Kanya.Su@chula.ac.th

Received 16 February 2013; revised 27 April 2013; accepted 1 May 2013; published online 30 May 2013 
fluorescence was measured with a Cecil spectrofluorometer using 450-nm emission and 365-nm excitation filters. The normal range of enzyme activity was determined in leukocytes of six normal Thais. Each patient's major clinical features and enzymatic activity are shown in Table 1.

\section{Genotyping}

After informed consent was obtained, high-molecular weight genomic DNA of all patients except patient 1 was extracted from peripheral blood leukocytes. Long-template PCR amplifying the entire GBA gene was performed using the Elongase Enzyme Mix (Invitrogen, Carlsbad, CA, USA) with primers GBA-F1 and GBA-R11 (Table 2). DNA was denatured for $30 \mathrm{~s}$ at $94^{\circ} \mathrm{C}$, followed by 35 cycles of denaturation at $94{ }^{\circ} \mathrm{C}$ for $30 \mathrm{~s}$, annealing at $55^{\circ} \mathrm{C}$ for $30 \mathrm{~s}$ and elongation at $68^{\circ} \mathrm{C}$ for $7 \mathrm{~min}$. The $6.6-\mathrm{kb}$ product amplified by long-template PCR was gel purified using the QIAquick gel extraction kit (Qiagen, Valencia, CA, USA), then treated with ExoSAP-IT (USB Corporation, Cleveland, $\mathrm{OH}$ USA), and sent for direct sequencing at Macrogen Inc., Seoul, Korea. In addition, the genomic DNA of all patients including patient 1, whose highmolecular weight DNA was not available, was subjected to PCR amplification using primers designed to amplify each exon of the functional GBA gene but not the pseudogene (Table 2). PCR products were treated with ExoSAP-IT and sent for direct sequencing. Sequence analyses were performed by Sequencher 4.2. Samples with possible new variants were resequenced. The positions of mutations were compared with the GBA coding sequence (GenBank accession no. J03059.1)

The p.L483P (L444P), p.Y402H and p.X537A mutations were verified by restriction enzyme digestions of the patients' PCR products, using NciI, RsaI and Cac8I, respectively. Parents of patients with probable homozygous mutations for the p.L483P (L444P) mutation were also examined for the variants by restriction enzyme analysis. RNA from patient 5 who had the IVS6 mutation was extracted from peripheral blood leukocytes using QIAamp RNA blood mini kit (Qiagen). Reverse transcription was performed using ImProm-II reverse transcriptase (Promega, Madison, WI, USA) according to company recommendations. PCR amplification of the GBA cDNA covering the $3^{\prime}$-end of exon 4 through the termination codon to the $5^{\prime}$ - end of exon 11 was performed using primers cGBA4 and cGBA-R11 designed to amplify only the functional GBA cDNA, but not the cDNA of the pseudogene (Table 2).

\section{Haplotype analysis of the p.L483P (L444P) allele}

We used PCR-RFLP to detect the p.L483P (L444P) mutation in four additional patients with GD. Three were homozygous and one heterozygous for the mutation. DNA of these four cases, patients 1,2 and 3, and available parents were PCR-amplified with primers as shown in Table 3 for previously described microsatellites (5GC3.2, ITG6.2, D1S2777, D1S1595 D1S2721). DNA of patient 4 was not available for this haplotype analysis. Genotyping was performed by

Table 1 Clinical, biochemical and molecular data of the Thai GD patients

Genotype $^{\mathrm{C}}$

\begin{tabular}{|c|c|c|c|c|c|c|}
\hline \multirow[b]{2}{*}{ Patient no. } & \multirow[b]{2}{*}{ Sex } & \multirow[b]{2}{*}{$\begin{array}{l}\text { Age at last follow-up } \\
\text { or death }\end{array}$} & \multirow[b]{2}{*}{ Type of GD; clinical features ${ }^{\mathrm{a}}$} & \multirow[b]{2}{*}{ Enzyme activity ${ }^{b}$} & \multicolumn{2}{|c|}{ Genotype $^{c}$} \\
\hline & & & & & DNA mutation & Protein change \\
\hline 1 & M & 18 months & $\begin{array}{l}\text { 2; HS at age } 7 \text { months, regression of develop- } \\
\text { ment, and death at age } 18 \text { months }\end{array}$ & NA & c. $1448 \mathrm{~T}>\mathrm{C} / \mathrm{c} .681 \mathrm{~T}>\mathrm{C}$ & $\begin{array}{l}\text { p.L483P (L444P)/ } \\
\text { p.N227K (N188K) }\end{array}$ \\
\hline 2 & $\mathrm{~F}$ & 16 years & $\begin{array}{l}1 ; \mathrm{HS} \text { at age } 3 \text { years, pancytopenia, splenect- } \\
\text { omy at age } 7 \text { years. Cirrhosis and hepatic fail- } \\
\text { ure, leading to death at age } 16 \text { years }\end{array}$ & 1.07 & c. $1448 \mathrm{~T}>\mathrm{C} / \mathrm{c} .1448 \mathrm{~T}>\mathrm{C}$ & p.L483P/p.L483P \\
\hline 3 & $\mathrm{~F}$ & 12 years & $\begin{array}{l}1 \text {; HS at age } 1 \text { year, anemia and thrombocyto- } \\
\text { penia, splenectomy at age } 4 \text { years, chronic bone } \\
\text { pain, and a pathologic fracture of right femoral } \\
\text { neck at age } 9 \text { years. She died from sepsis at age } \\
12 \text { years }\end{array}$ & 0 & c. $1448 \mathrm{~T}>\mathrm{C} / \mathrm{c} .1448 \mathrm{~T}>\mathrm{C}$ & p.L483P/p.L483P \\
\hline 4 & $\mathrm{~F}$ & 2 years & $\begin{array}{l}\text { 1; HS at age } 7 \text { months, pancytopenia, sple- } \\
\text { nectomy at age } 17 \text { months }\end{array}$ & 0.62 & c. $1448 \mathrm{~T}>\mathrm{C} / \mathrm{c} .1204 \mathrm{~T}>\mathrm{C}$ & p.L483P/p.Y402H \\
\hline
\end{tabular}

Abbreviations: F, female; GD,Gaucher disease; HS, hepatosplenomegaly; M, male; NA, not available.

aAll patients had HS and Gaucher cells in aspirated bone marrow. None received enzyme replacement therapy or bone marrow transplantation.

bEnzyme activity is expressed in $\mathrm{nmol} \mathrm{mg}{ }^{-1}$ protein per $\mathrm{h}$, with the values of six Thai normal controls being $11.63 \pm 4.97$ (range: 5.97-19.58).

cNovel mutations are indicated in bold.

${ }^{\mathrm{d}} \mathrm{L} 444 \mathrm{P}$ and N188K are the names that follow the common nomenclature and do not include the 39-residue signal peptide.

Table 2 PCR primers used to amplify genomic and complementary DNA of GBA

\begin{tabular}{|c|c|c|}
\hline Gene/exon amplified & Primer sequence ( $5^{\prime}$ to $\left.3^{\prime}\right)$ & Product size (bp) \\
\hline g. $G B A$ and its pseudogene & GBA-F1: 5'-CCT AAA GTT GTC ACC CAT AC-3' GBA-R11: 5'-ACC ACC TAG AGG GGA AAG TG-3' & 6.6 and $4 \mathrm{~kb}$ \\
\hline g. exons $1-2$ & GBA-F1: 5'-CCT AAA GTT GTC ACC CAT AC-3' GBA-R2: 5'-CCA CCG AGC TGT AGC C-3' & 1382 \\
\hline g. exons 3-4 & GBA-F34: 5'-ATG TGT CCA TTC TCC ATG TCT TCA-3' GBA-R34: 5’-ACG AAA AGT TTC CGT GGC TCT-3' & 523 \\
\hline g. exons $5-6$ & GBA-F56: 5'-GAC CTC AAA TGA TAT ACC TG-3' GBA-R56: 5'-GAA AGG TCA TGA ATG A-3' & 1690 \\
\hline g. exon 7 & GBA-F7: 5'-TCA AGA CCA ATG GAG CGG TG-3' GBA-R7: 5'-AGT TTG GGA GCC AGT CAT TT-3' & 953 \\
\hline g. exon 8 & GBA-F8: 5'-GTT GCA TTC TTC CCG TCA CC-3' GBA-R8: 5'-CTG GAC AGG AAG GGC TTC TG-3' & 367 \\
\hline g. exons 9-11 & GBA-F911: 5'-AAC CAT GAT TCC CTA TCT TC-3' GBA-R911: 5'-ACC ACC TAG AGG GGA AAG TG-3' & 1249 \\
\hline c. 3 -end of exon 4 to $5^{\prime}$-end of exon 11 & cGBA4: 5'-TGA CAG ATG CTG CTG CTC TC-3' GBA-R11: 5'-ACC ACC TAG AGG GGA AAG TG-3' & 1409 \\
\hline c. entire coding region of the $\mathrm{X} 537 \mathrm{~A}$ mutant & GBA-Xhol-F: 5'-ATC TCG AGC ATC TAA TGA CC-3'GBA-EcoRI-R: 5'-ATG AAT TCT GAG TCAC CCA A-3' & 1797 \\
\hline
\end{tabular}

Abbreviations: c, complementary DNA; g, genomic DNA; GBA, glucocerebrosidase. 
Macrogen. Data were analyzed by GeneScan software (Applied Biosystem, Carlsbad, CA, USA).

\section{Functional analysis of the p.X537A mutation}

For glucocerebrosidase activity analysis, RNA of normal controls and patient 5 was extracted from peripheral blood leukocytes with QIAamp RNA blood mini kit (Qiagen), then reverse-transcribed and amplified by PCR using primers GBA-XhoI-F and GBA-EcoRI-R (Table 2) to add the XhoI and EcoRI sites at the $5^{\prime}$ and $3^{\prime}$ ends, respectively. The amplified cDNA was cloned into the pGEM-T Easy Vector System (Promega) and subsequently into the pcDNA3.1 expression vector (Invitrogen), and sequenced. These constructs were transiently transfected into COS-7 (fibroblast) cells, using Lipofectamine 2000 (Invitrogen). After 24-hr transfection, cells were centrifuged and sonicated in $\mathrm{dH}_{2} \mathrm{O}$. Protein concentration was determined by BCA protein assay (Micro BCA Protein Assay Kit, PIERCE, Rockford, IL, USA) and the glucocerebrosidase activity was measured by fluorimetric assay using 4-methyl-umbelliferone- $\beta$-D-glucopyranoside.

For protein expression analysis, the p.N409S (N370S) mutation, a positive control for the enzyme mislocalization to the endoplasmic reticulum (ER), ${ }^{4}$ was generated using QuikChange Site-Directed Mutagenesis Kit (Stratagene, La Jolla, CA, USA). The GBA clones were confirmed by direct sequencing. Cells transfected with the wild-type, p.X537A and p.N409S (N370S) GBA were immunoblotted to measure the level of glucocerebrosidase. Protein extracts were prepared from cell pellets harvested in lysis buffer with freshly added protease inhibitors, subjected to $10 \%$ polyacrylamide gel electrophoresis and

Table 3 Markers and primers to determine haplotypes

\begin{tabular}{|c|c|c|}
\hline Marker & Forward primer ( $5^{\prime}$ to $\left.3^{\prime}\right)$ & Reverse primer $\left(5^{\prime}\right.$ to $\left.3^{\prime}\right)$ \\
\hline D1S2777 & 5'-GCACCACGGAACTCCAGTAT-3' & 5'-CACCACTGTGCCCAGCTAAT-3' \\
\hline D1S2721 & 5'-TTGCTCGGCCAGAGTCT-3' & 5'-ACGCATCACACCTGGCTAGT-3' \\
\hline D1S1595 & 5'-ATGGTATGAACCTGGAGGTG-3' & 5'-GGCAGATAAAAGGACTGCAA-3' \\
\hline ITG6.2 & 5'-CACATGAGGTCAGGTGTTTG-3' & 5'-GCAAAGGAGTGGTGAACTTC-3' \\
\hline $5 \mathrm{GC} 3.2$ & 5'-TTCAATCGCCCCCATCCACC- $3^{\prime}$ & 5'-TCAGAGCCCTTCCTCAAG-3' \\
\hline
\end{tabular}

transferred to PVDF membranes. The membranes were blotted with antibodies to glucocerebrosidase (Santa Cruz Biotechnology, Santa Cruz, CA, USA) and actin (Santa Cruz Biotechnology), a loading control.

For subcellular localization analysis, the COS-7 cells transfected with the wild-type and p.X537A GBA were analyzed by immunofluorescence staining. Cells were grown directly on Lab-Tek II chamber slides, and fixed $24-48 \mathrm{~h}$ after transfection with cold methanol. Cells were incubated with rabbit polyclonal immunoglobulin G antibodies to glucocerebrosidase (Santa Cruz Biotechnology), and mouse monoclonal immunoglobulin G1 antibodies to LAMP2 as a lysosomal marker (Abcam, Cambridge, UK). These cells were subsequently analyzed with Carl Zeiss LSM 700 confocal microscope (Jena, Germany).

\section{RESULTS}

The clinical, biochemical and mutational profiles of patients are summarized in Table 1. Four patients were classified as GD type 1 and one as type 2. Among patients with type $1 \mathrm{GD}$, the onset of hepatosplenomegaly ranged from 7 months to 7 years old. All patients whose leukocytic glucocerebrosidase activities were determined had no or markedly low activities $\left(<1.07 \mathrm{nmol} \mathrm{mg}{ }^{-1}\right.$ protein per hour) compared with $11.63 \pm 4.97 \mathrm{nmol} \mathrm{mg}^{-1}$ protein per hour in Thai controls.

We successfully identified all 10 mutant alleles with either long-template PCR or PCR using specific primers for the functional GBA gene (Table 2). Both methods gave the same results. Four patients had the p.L483P (L444P) mutation. Patients 1 and 4 were compound heterozygotes (Figure la lower panel, lanes 5 and 6, respectively). Patients 2 and 3 were homozygotes (Figure 1a lower panel, lanes 4 and 3, respectively). The parents of patients 2 and 3 denied consanguinity, and were all heterozygous for the c.1448T >C (p.L483P) mutation. Patient 2 died from hepatic failure at age 16 years. Patient 3 had thrombocytopenia requiring splenectomy at age 4, fracture of her right femoral neck at age 9 and died from sepsis at age a

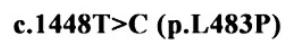
AACGACCTGGACGCA

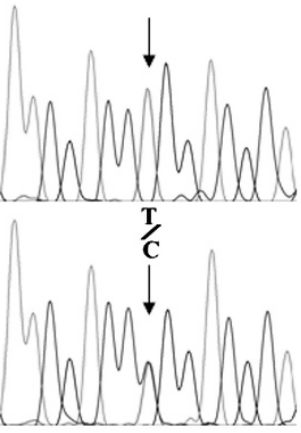

M123456789M

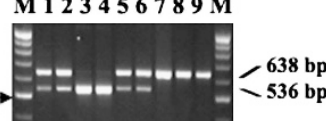

b

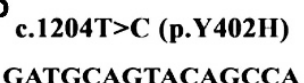

GATGCAGTACAGCCA

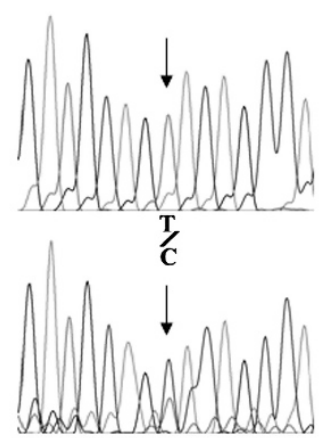

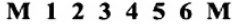

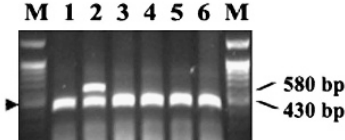

C

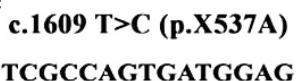

TCGCCAGTGATGGAG
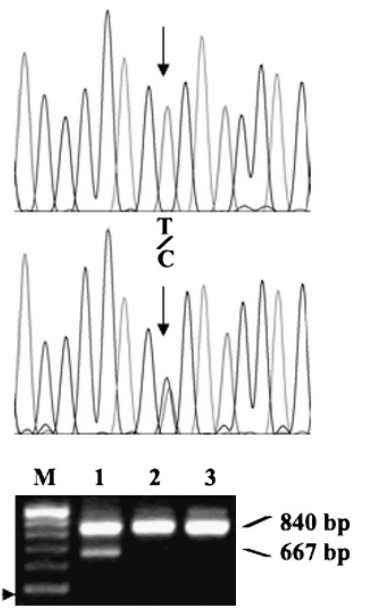

Figure 1 Mutation analysis of the common p.L483P (L444P) mutation (column a) and the novel mutations, p.Y402H (column b) and p.X537A (column c). Upper and middle panels, electropherograms of the wild-type and heterozygous mutant alleles, respectively. Lower panel, restriction enzyme digestion analysis. $\mathrm{M}$ is a 100-bp marker. The arrow heads indicate the 500-bp bands. In column a, the p.L483P (L444P) mutation creates an Ncil restriction site. Therefore, the 638-bp PCR products of the mutant allele of individuals homozygous (lanes 3 and 4 ) and heterozygous (lanes 1 , 2, 5, and 6) for the mutation were digested into 536-bp and 102-bp (not shown) products. In column b, the p.Y402H mutation in patient 4 (lane 2) eliminates the Rsal site, leaving the 580-bp product intact. The 580-bp product was digested into 430 and 150 -bp (not shown) products of the other allele. In column c, the p.X537A mutation in patient 5 (lane 1) creates a Cac8l site. Therefore, the 840-bp product of the mutant allele was digested into 667 and $173-$ bp (not shown) products. A full color version of this figure is available at the Journal of Human Genetics journal online. 
Table 4 Forms and frequencies of haplotypes of the p. L483P (L444P) mutation in Thai patients

\begin{tabular}{|c|c|c|c|c|c|c|}
\hline Patient & Mutation & ITG6.2 & $5 G C 3.2$ & D1S2777 & D1S1595 & D1S2721 \\
\hline \multirow[t]{2}{*}{1} & p.L483P & 314 & 219 & 264 & 279 & 232 \\
\hline & p.N227K & 314 & 221 & 264 & 279 & 236 \\
\hline \multirow[t]{2}{*}{2} & p. L483Pa & 318 & 217 & 268 & 279 & 238 \\
\hline & p.L483Pa & 318 & 217 & 268 & 279 & 238 \\
\hline \multirow[t]{2}{*}{3} & p.L483Pa & 318 & 217 & $266 / 270$ & $279 / 283$ & $234 / 236$ \\
\hline & p.L483P & 314 & 219 & & & \\
\hline \multirow[t]{2}{*}{4} & p.L483P & ND & ND & ND & ND & ND \\
\hline & p.Y402H & ND & ND & ND & ND & ND \\
\hline \multirow[t]{2}{*}{6} & p.L483Pc & 306 & 217 & 268 & 279 & 240 \\
\hline & p.L483Pc & 306 & 217 & 268 & 279 & 240 \\
\hline \multirow[t]{2}{*}{7} & p.L483Pa & 318 & 217 & 268 & 283 & 238 \\
\hline & p.L483Pa & 318 & 217 & 268 & 283 & 238 \\
\hline \multirow[t]{2}{*}{8} & p.L483Pd & 322 & 217 & 268 & 279 & 238 \\
\hline & p.L483Pb & 314 & 219 & 264 & 279 & 232 \\
\hline \multirow[t]{2}{*}{9} & p.L483Pa & 318 & 217 & 268 & 279 & 238 \\
\hline & $?$ & 314 & 221 & 264 & 279 & 236 \\
\hline
\end{tabular}

\begin{tabular}{llc} 
& $n$ & Haplotype frequency \\
\hline Haplotype a & 6 & 0.50 \\
Haplotype b & 3 & 0.25 \\
Haplotype c & 2 & 0.17 \\
Haplotype d & 1 & 0.08 \\
\hline
\end{tabular}

Abbreviation: ND, not determined; ?, unidentified.

L483P (L444P) mutation in Thai patients.

12. This p.L483P (L444P) mutation was found in four different haplotypes (Table 4).

Two novel mutations were identified. A novel non-synonymous variant, c. $1204 \mathrm{~T}>\mathrm{C}$ (p.Y402H), in exon 8 was found in patient 4 (Figure $1 \mathrm{~b}$ middle panel and lower panel) and a novel termination codon mutation, c.1609T >C (p.X537A) (Figure 1c middle panel and lower panel), was found in patient 5 . The boy was heterozygous for a splice site mutation, IVS6-1G >C. Sequencing of his cDNA revealed only the sequence of the p.X537A allele with no detectable level of the IVS6-1G $>C$ transcript (Figure 1c lower panel). Expression of the p.X537A mutant and the wild-type clones in COS-7 cells demonstrated that the mutation reduced the residual glucocerebrosidase enzyme activity with respect to controls (Figure 2) and reduced the protein expression level (Figure 3), but did not significantly change the protein subcellular localization in mammalian cells (Figure 4).

\section{DISCUSSION}

The presence of a highly homologous pseudogene sequence and several types of mutations including large recombinant alleles or deletions has made mutation analysis of GBA challenging. ${ }^{5}$ Using solely conventional PCR and single-strand conformation polymorphism (SSCP) or PCR-based mutation-detection techniques to screen for specific mutations might result in a significant number of unidentified mutant alleles. ${ }^{6}$ Therefore, in this study, direct mutation detection was performed by sequencing the entire coding region in all patients either by long-template PCR or PCR using specific primers for the functional gene. With these methods, all ten mutant alleles were identified in our five patients (see Table 1).

Patient 1 was compound heterozygous for p.L483P/p.N227K (L444P/N188K). The N188K mutation is a rare mutation previously found in patients with type 2 GD. ${ }^{7}$ Sequencing electropherogram of

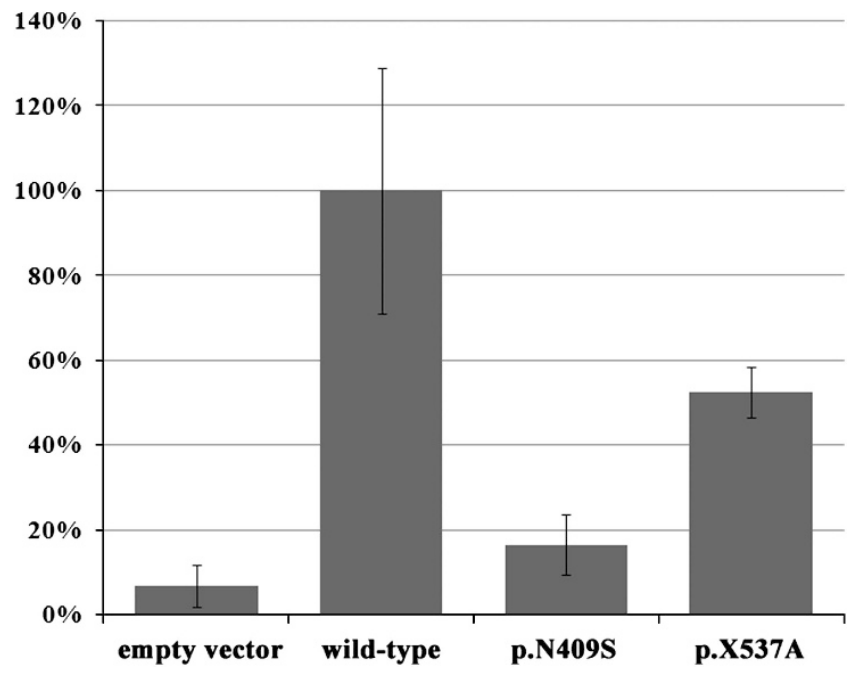

Figure 2 Glucocerebrosidase (GBA) activity analysis of the p.X537A mutant. Enzyme activity was measured on extracts of cells transfected with the empty pcDNA3.1 vector (empty vector), wild-type GBA (wild-type), p.N409S mutant (p.N409S) and p.X537A mutant (p.X537A). Enzyme activity from cells transfected with the wild-type $G B A$ was used as a baseline.

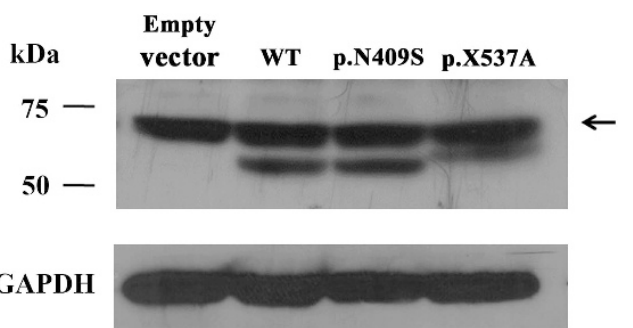

Figure 3 Glucocerebrosidase (GBA) expression analysis of p.X537A by western blotting. Top panel: Empty vector, wild-type (WT), p.N409S, p.X537A $=$ cells transfected with the empty pcDNA3.1 vector, wild-type GBA, p.N409S mutant and p.X537A mutant, respectively. Arrow =a nonspecific band. Lower panel: glyceraldehyde 3-phosphate dehydrogenase (GAPDH) loading control.

patients 2 and 3 showed only the c.1448T $>$ C (p.L483P) mutation, which could be homozygous or hemizygous. Therefore, the genotypes of their parents were studied and revealed that all were heterozygous for the p.L483P (L444P) mutation, suggesting that patients 2 and 3 were homozygous for the mutation. p.L483P (L444P) is the most common mutant allele in the Thai patients with GD studied, accounting for $60 \%$ of mutant alleles. Notably, because conventional PCR method could not distinguish the isolate p.L483P (L444P) mutation and the p.L483P (L444P) as a part of a recombinant allele carrying additional pseudogene sequence alterations, further evaluation by other techniques such as direct sequencing to establish the presence or absence of a complex allele should be considered. ${ }^{8,9}$ The p.L483P (L444P) mutation occurs with significant frequencies in various populations, including $41 \%$ of mutant alleles in the Japanese, ${ }^{6}$ and $2.84 \%$ in the Jewish. ${ }^{10}$ At the age of death at 16 years in patient 2 and 12 years in patient 3, both patients with homozygous p.L483P (L444P) did not show primary neurological manifestations, and, therefore, were categorized as type 1. A previously reported Thai patient with homozygous for the p.L483P (L444P) mutation was described to have a 'Norbottnian-like' 

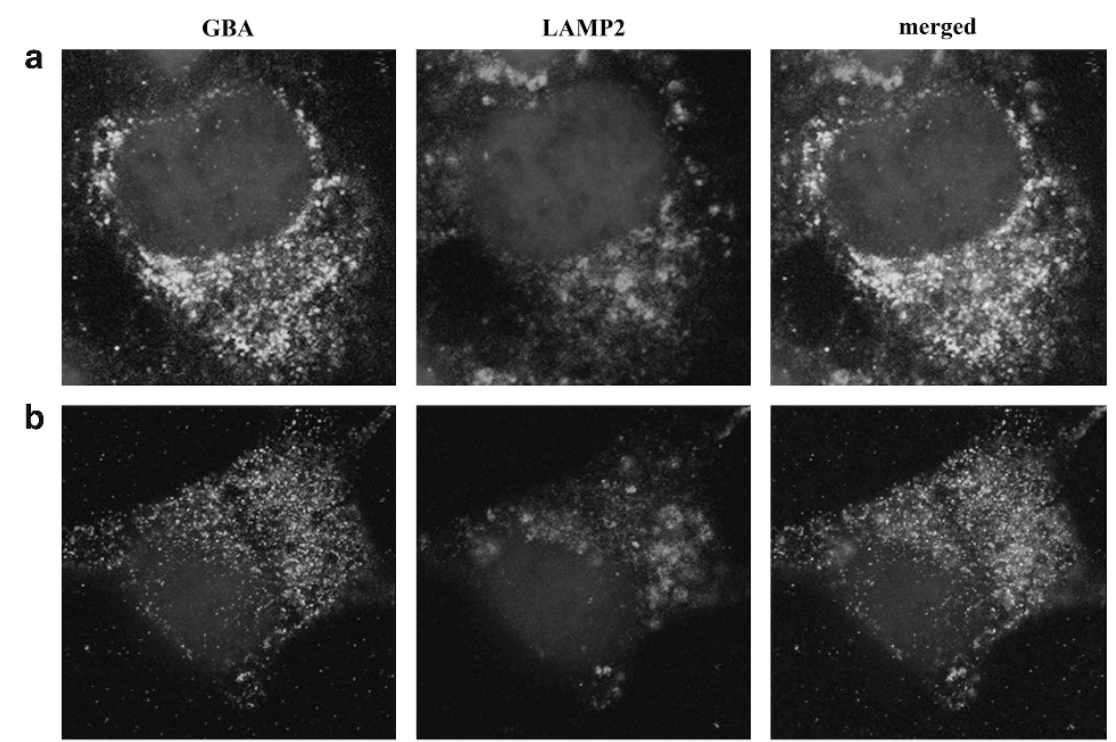

C
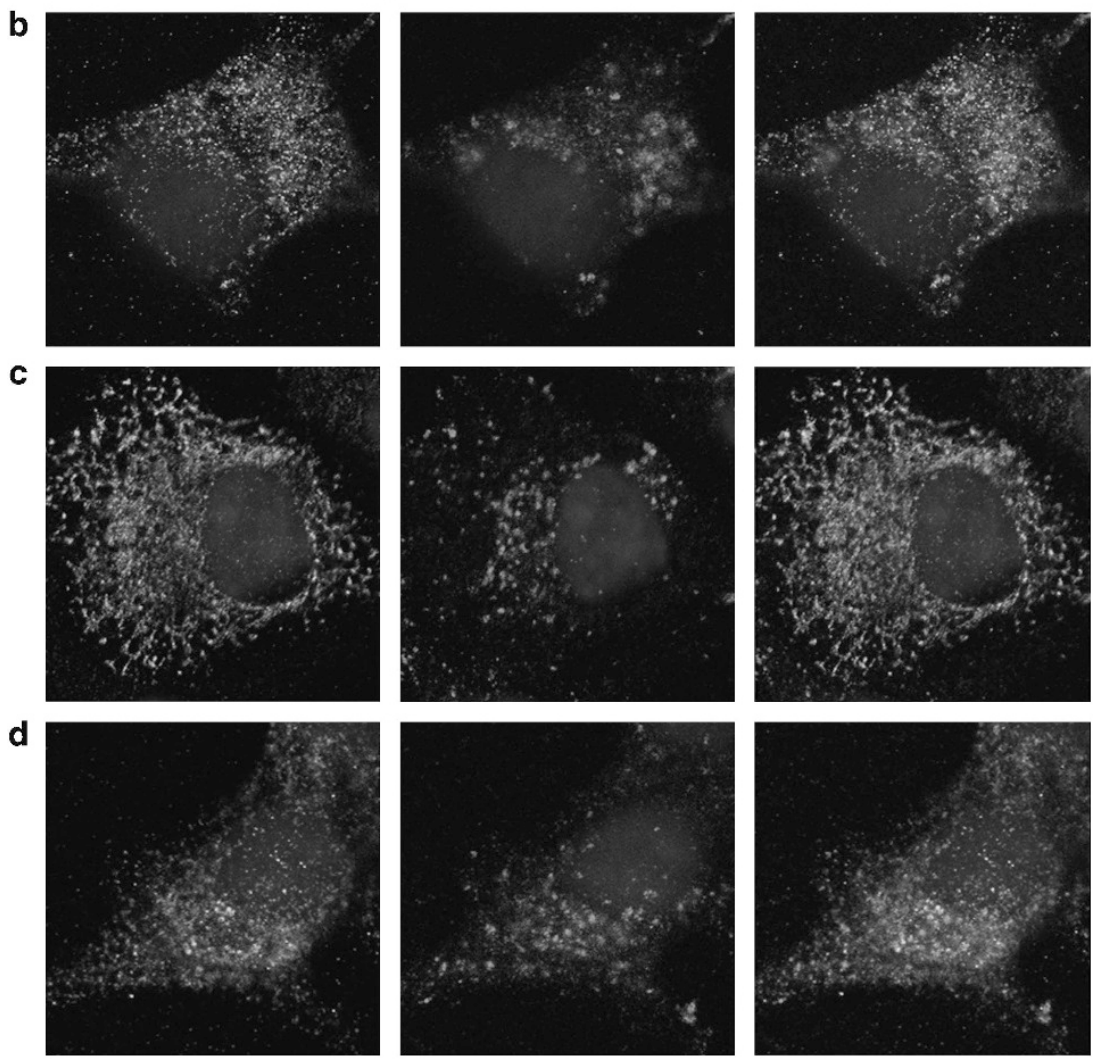

e
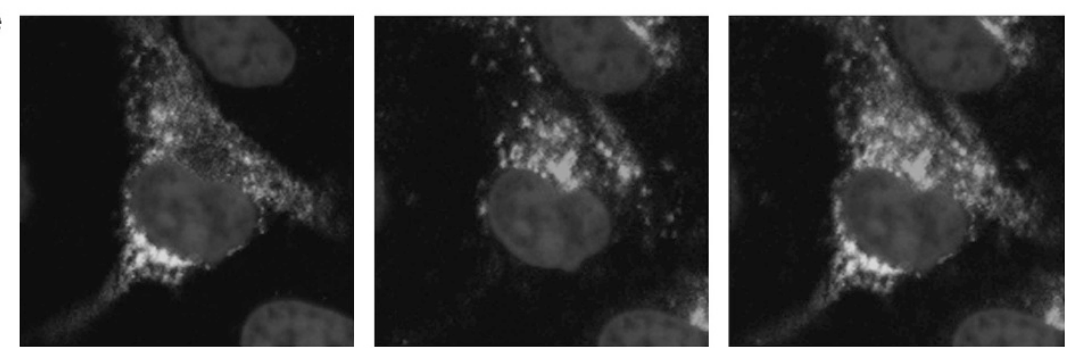

Figure 4 Subcellular localization analysis of p.X537A mutant by immunofluorescence confocal microscopy. (a, b)=wild-type glucocerebrosidase (GBA), (c, d) $=$ p.X537A mutant, $E=$ p.N409S. Green $=$ GBA, red $=$ LAMP2 (a lysosomal marker), blue = nuclei counterstained with 4',6-diamidino-2-phenylindole. A full color version of this figure is available at the Journal of Human Genetics journal online.

phenotype, with mild neurological involvement. ${ }^{11}$ However, 15 of 19 Caucasian patients homozygous for the p.L483P (L444P) had neuronopathic GD. ${ }^{12}$ In addition, 9 of 12 Japanese patients with this genotype who were originally diagnosed with non-neuronopathic GD, developed neurological signs/symptoms during follow-up. ${ }^{13}$ Patient 2 died from cirrhosis and hepatic failure. Owing to no other identifiable causes of cirrhosis, this could be a complication of GD. There are several previous reports of GD patients dying from cirrhosis. ${ }^{14,15}$ This p.L483P (L444P) mutation was found in four different haplotypes, suggesting that it was a recurrent mutation, not caused by a founder effect.

Two novel mutations including a missense (c.1204T > C, p.Y402H) and a mutation in the termination codon (c.1609T $>$ C, p.X537A) were identified. A splice site mutation previously reported in a Thai patient with type $3 \mathrm{GD},{ }^{11}$ the IVS6-1G $>\mathrm{C}$ change, was compound

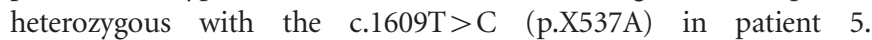
Sequencing of his cDNA showed no skipping around IVS6 and only the $\mathrm{C}$ at position 1609 (Figure 1c lower panel), suggesting that it 
contained only the sequence of the p.X537A allele. It is probable that the IVS6-1G $>C$ is pathogenic due to the absence of its mRNA, which is most likely from a splicing defect, leading to a premature stop codon and degradation of its mRNA via a nonsense mediated mRNA decay. ${ }^{16}$ The c.1609T $>C$ (p.X537A) mutation is the first identified termination codon mutation in the GBA gene and expected to result in the addition of 15 more amino acids before coming to a new stop. Functional studies have suggested that the mutation decreases the amount of protein being expressed and the enzymatic activity, while it is still correctly localized. Termination codon mutations have been observed in many disorders, including Wiskott-Aldrich syndrome. ${ }^{17}$

In conclusion, we report five Thai patients with GD. A common mutation, p.L483P (L444P), accounting for $60 \%$ of mutant alleles, and two novel mutations, c.1204T $>$ C (p.Y402H) and c.1609T $>C$ (p.X537A), were identified. Phenotypes of the two patients homozygous for p.L483P (L444P) were severe; both died in their teens. Long-term follow-up is necessary in determining the disease severity associated with the novel mutations.

\section{CONFLICT OF INTEREST}

The authors declare no conflicts of interest.

\section{ACKNOWLEDGEMENTS}

We would like to thank Dr Darintr Sosothikul for referring patients to us and Dr Nipan Israsena for technical advices. This study was supported by Ratchadaphiseksomphot Endowment Fund, Faculty of Medicine, Chulalongkorn University, the Thailand Research Fund, and The Integrated Innovation Academic Center, Chulalongkorn University Centenary Academic Development Project (CU56-HR05), and the Higher Education Research Promotion and National Research University Project of Thailand, Office of the Higher Education Commission (HR1163A). Duangrurdee Wattanasirichaigoon is a recipient of Research Career Development Awards from the Faculty of Medicine, Ramathibodi Hospital, Mahidol University.
1 Grabowski, G. A. Recent clinical progress in Gaucher disease. Curr. Opin. Pediatr. 17, 519-524 (2005).

2 Horowitz, M., Wilder, S., Horowitz, Z., Reiner, O., Gelbart, T. \& Beutler, E. The human glucocerebrosidase gene and pseudogene: structure and evolution. Genomics 4, 87-96 (1989).

3 Diaz, G. A., Gelb, B. D., Risch, N., Nygaard, T. G., Frisch, A., Cohen, I. J. et al. Gaucher disease: the origins of the Ashkenazi Jewish N370S and 84GG acid beta-glucosidase mutations. Am. J. Hum. Genet. 66, 1821-1832 (2000).

4 Schmitz, M., Alfalah, M., Aerts, J. M., Naim, H. Y. \& Zimmer, K. P. Impaired trafficking of mutants of lysosomal glucocerebrosidase in Gaucher's disease. Int. J. Biochem. Cell Biol. 37, 2310-2320 (2005).

5 Tayebi, N., Stubblefield, B. K., Park, J. K., Orvisky, E., Walker, J. M., LaMarca, M. E. et al. Reciprocal and nonreciprocal recombination at the glucocerebrosidase gene region: implications for complexity in Gaucher disease. Am. J. Hum. Genet. 72, 519-534 (2003).

6 Eto, Y. \& Ida, H. Clinical and molecular characteristics of Japanese Gaucher disease. Neurochem. Res. 24, 207-211 (1999).

7 Stone, D. L., Tayebi, N., Orvisky, E., Stubblefield, B., Madike, V. \& Sidransky, E. Glucocerebrosidase gene mutations in patients with type 2 Gaucher disease. Hum. Mutat. 15, 181-188 (2000).

8 Koprivica, V., Stone, D. L., Park, J. K., Callahan, M., Frisch, A., Cohen, I. J. et al. Analysis and classification of 304 mutant alleles in patients with type 1 and type 3 Gaucher disease. Am. J. Hum. Genet. 66, 1777-1786 (2000).

9 Tayebi, N., Stern, H., Dymarskaia, I., Herman, J. \& Sidransky, E. 55-base pair deletion in certain patients with Gaucher disease complicates screening for common Gaucher alleles. Am. J. Med. Genet. 66, 316-319 (1996).

10 Grabowski, G. A. Gaucher disease: gene frequencies and genotype/phenotype correlations. Genet. Test. 1, 5-12 (1997).

11 Suwannarat, P., Keeratichamroen, S., Wattanasirichaigoon, D., Ngiwsara, L., Cairns, J. R., Svasti, J. et al. Molecular characterization of type 3 (neuronopathic) Gaucher disease in Thai patients. Blood Cells Mol. Dis. 39, 348-352 (2007).

12 Sidransky, E., Tsuji, S., Martin, B. M., Stubblefield, B. \& Ginns, E. I. DNA mutation analysis of Gaucher patients. Am. J. Med. Genet. 42, 331-336 (1992).

13 Ida, H., Rennert, O. M., Iwasawa, K., Kobayashi, M. \& Eto, Y. Clinical and genetic studies of Japanese homozygotes for the Gaucher disease L444P mutation. Hum. Genet. 105, 120-126 (1999).

14 Ida, H., Rennert, O. M., Ito, T., Maekawa, K. \& Eto, Y. Clinical and genetic studies of five fatal cases of Japanese Gaucher disease type 1. Acta. Paediatr. Jpn. 38, 233-236 (1996).

15 Perel, Y., Bioulac-Sage, P., Chateil, J. F., Trillaud, H., Carles, J., Lamireau, T. et al. Gaucher's disease and fatal hepatic fibrosis despite prolonged enzyme replacement therapy. Pediatrics 109, 1170-1173 (2002).

16 Conti, E. \& Izaurralde, E. Nonsense-mediated mRNA decay: molecular insights and mechanistic variations across species. Curr. Opin. Cell. Biol. 17, 316-325 (2005).

17 Chatchatee, P., Srichomthong, C., Chewatavorn, A. \& Shotelersuk, V. A novel termination codon mutation of the WAS gene in a Thai family with Wiskott-Aldrich syndrome. Int. J. Mol. Med. 12, 939-941 (2003). 